\title{
Relationship Between Post-exercise Heart Rate Recovery and Changing Ratio of Cardiopulmonary Exercise Capacity
}

\author{
Ji-Hyun Kim, $\mathrm{MD}^{1}$, Yu-Ri Choe, $\mathrm{MD}^{2}$, Min-Keun Song, $\mathrm{MD}^{1}$, \\ In-Sung Choi, $\mathrm{MD}, \mathrm{PhD}^{1}$, Jae-Young Han, $\mathrm{MD}, \mathrm{PhD}^{1}$ \\ ${ }^{1}$ Department of Physical and Rehabilitation Medicine, Regional Cardiocerebrovascular Rehabilitation Center- \\ Research Institute of Medical Sciences, Chonnam National University Medical School \& Hospital, Gwangju; \\ ${ }^{2}$ Department of Physical and Rehabilitation Medicine, Gwangju Veterans Hospital, Gwangju, Korea
}

\begin{abstract}
Objective To determine whether heart rate recovery (HRR) following an exercise tolerance test (ETT) is correlated with a changing ratio of peak oxygen consumption $\left(\mathrm{VO}_{2}\right)$ and maximal metabolic equivalents $\left(\mathrm{MET}_{\max }\right)$.

Methods A total of 60 acute myocardial infarction (AMI) patients who underwent ETT at both assessment points - 3 weeks (T0) after the AMI attack and 3 months after T0 (T1) were included. After achieving a peak workload, the treadmill was stopped with a 5-minute cooldown period, and the patients recovered in a comfortable and relaxed seated position. HRR was defined as the difference between the maximal heart rate $\left(\mathrm{HR}_{\max }\right)$ and the $\mathrm{HR}$ measured at specific time intervals - immediately after the cool down period (HRR-0) and 3 minutes after the completion of the ETT (HRR-3).

Results HRR-0 and HRR-3 increased over time, whereas $\mathrm{VO}_{2 \max }$ and $\mathrm{MET}_{\max }$ did not show significant changes. There was a positive correlation between HRR at T0 and the exercise capacity at T0. HRR at T0 also showed a positive correlation with the exercise capacity at $\mathrm{T} 1$. There was no significant correlation between HRR measured at $\mathrm{T} 0$ and the change in the ratio of $\mathrm{VO}_{2 \max }$ and $\mathrm{MET}_{\text {max }}$, as calculated by subtracting $\mathrm{VO}_{2 \max }$ and $\mathrm{MET}_{\max }$ obtained at $\mathrm{T} 0$ from those obtained at $\mathrm{T} 1$, divided by $\mathrm{VO}_{2 \max }$ at $\mathrm{T} 0$ and multiplied by 100 .

Conclusion Post-exercise HRR measured at 3 weeks after the AMI onset can reflect the exercise capacity 3 months after the first ETT. However, it may be difficult to correlate post-exercise HRR at T0 with the degree of increase in cardiopulmonary exercise capacity in patients with AMI.
\end{abstract}

Keywords Heart rate, Myocardial infarction, Exercise tolerance, Exercise test, Autonomic nervous system

Received March 8, 2017; Accepted May 2, 2017

Corresponding author: Jae-Young Han

Department of Physical and Rehabilitation Medicine, Chonnam National University Medical School \& Hospital, 42 Jebong-ro, Dong-gu, Gwangju 61469, Korea. Tel: +82-62-220-5198, Fax: +82-62-228-5975, E-mail: rmhanjy@daum.net

ORCID: Ji-Hyun Kim (http://orcid.org/0000-0003-4892-6753); Yu-Ri Choe (http://orcid.org/0000-0001-5712-8724); Min-Keun Song (http://orcid. org/0000-0001-8186-5345); In-Sung Choi (http://orcid.org/0000-0003-3967-9201); Jae-Young Han (http://orcid.org/0000-0003-1672-8875).

() This is an open-access article distributed under the terms of the Creative Commons Attribution Non-Commercial License (http://creativecommons.org/ licenses/by-nc/4.0) which permits unrestricted noncommercial use, distribution, and reproduction in any medium, provided the original work is properly cited. Copyright $\odot 2017$ by Korean Academy of Rehabilitation Medicine 


\section{INTRODUCTION}

Cardiac autonomic dysfunction is commonly associated with cardiovascular disease, and it has been reported in subjects with risk factors for developing cardiovascular disease. Autonomic dysfunction in these populations is mainly characterized by an increase in sympathetic activity and withdrawal of parasympathetic activity. This autonomic imbalance increases cardiac load and ventricular instability, leading to an increased risk for the progression of heart disease and worse clinical outcomes [1]. Post-exercise heart rate recovery (HRR) provides a noninvasive and clinically feasible method to quantitatively assess parasympathetic function [2].

Several studies have shown that impaired HRR after exercise cessation is associated with poor clinical outcomes, including an increased risk of mortality in subjects referred for stress testing [2-12]. Youn et al. [11] showed that impaired post-exercise HRR independently predicts clinical outcomes in prospectively and consecutively enrolled acute decompensated heart failure patients. Some studies have reported an association of HRR with peak oxygen consumption $\left(\mathrm{VO}_{2 \max }\right)[9,13]$. $\mathrm{VO}_{2 \max }$ during a cardiopulmonary exercise test (CPET) is a powerful independent prognostic marker to risk stratify patients with heart disease, and it is also an objective measure of functional capacity [14-16]. Based on these reports, we hypothesized that HRR might also be related to the degree of increase in cardiopulmonary exercise capacity. However, few studies have examined whether HRR is correlated with increased cardiopulmonary exercise capacity expressed as the changing ratios of $\mathrm{VO}_{2 \max }$ and maximal metabolic equivalents $\left(\mathrm{MET}_{\max }\right)$ in populations with acute myocardial infarction (AMI).

Thus, the objective of this study was to determine if the HRR in the first exercise tolerance test (ETT) performed 3 weeks after AMI onset can predict not only the clinical outcomes but also the degree of improvement in functional capacity in patients with AMI.

\section{MATERIALS AND METHODS}

This retrospective study analyzed the medical records of 655 patients diagnosed with AMI (ST elevation and non-ST elevation myocardial infarction) between August 2014 and July 2016 who were referred to our rehabilita- tion center after undergoing percutaneous coronary intervention (PCI). Among the patients who underwent ETT at about 3 weeks after the onset of myocardial infarction (T0) and about 3 months after the completion of the first ETT (T1), the following were excluded: (1) those with an unstable medical condition, such as a recurrence or re-admission during cardiac rehabilitation, those who underwent cardiac surgery, and those with unstable musculoskeletal conditions such as severe pain, contracture, and paralysis; (2) those with high risk factors on risk classification (i.e., left ventricular ejection fraction [EF] $<40 \%$ ); and (3) those who participated in a center-based cardiac rehabilitation program. Ultimately, 60 patients (53 men, seven women) were included in this study.

This study was approved by the Chonnam National University Hospital Institutional Review Board (No. CNUH2017-031).

All patients were educated regarding the importance of the modification of risk factors and regular exercise at about 3 weeks and 3 months after the AMI onset. However, patients who participated in center-based cardiac rehabilitation programs were excluded because such patients exhibit a significant improvement in functional capacity due to exercise training, and this could introduce a confounding variable. All included patients performed a symptom-limited ETT using the Modified Bruce Protocol.

Study outcomes were estimated from the ETT at both aforementioned assessment points. The body mass index (BMI) and history of comorbid conditions were assessed prior to the ETT. Heart rate (HR), $\mathrm{VO}_{2}$, and METs recorded at baseline and during ETT using an integrated metabolic measurement system (TrueOne 2400 Metabolic System; Parvo-Medics, Sandy, UT, USA) were used as clinical variables. Resting $\mathrm{HR}\left(\mathrm{HR}_{\mathrm{rest}}\right)$, maximal $\mathrm{HR}\left(\mathrm{HR}_{\mathrm{max}}\right)$, resting systolic blood pressure $\left(\mathrm{SBP}_{\text {rest }}\right)$, resting diastolic blood pressure $\left(\mathrm{DBP}_{\text {rest }}\right)$, maximal $\mathrm{SBP}\left(\mathrm{SBP}_{\max }\right)$, and maximal DBP $\left(\mathrm{DBP}_{\max }\right)$ were estimated using an automatic blood pressure and a pulse monitor, and a subjective measure of the rate of the perceived exertion was recorded. Changing ratios of $\mathrm{VO}_{2 \max }$ and $\mathrm{MET}_{\max }$ were calculated by subtracting $\mathrm{VO}_{2 \max }$ and $\mathrm{MET}_{\max }$ obtained at T0 from those obtained at $\mathrm{T} 1$, dividing it by the $\mathrm{VO}_{2 \max }$ at T0, and multiplying by 100 .

After achieving a peak workload, the treadmill slowed down the slope and speed for 5 minutes and then stopped. The patients recovered in a comfortable and relaxed seated position after a 'cool down' period. HRR was 
defined as the difference from $\mathrm{HR}_{\max }$ to the HR measured at specific time intervals - immediately after the cool down period (HRR-0) and 3 minutes after the completion of ETT (HRR-3).

Data were statistically analyzed with IBM SPSS Statistics ver. 22.0 (IBM, Armonk, NY, USA). We used a paired t-test to compare the categorical variables. The Pearson correlation coefficient and multivariate regression analysis were used to establish a link between the changes in the ratio of functional capacity and HRR. For the statistical tests, a p-value $<0.05$ was considered to be significant.

\section{RESULTS}

\section{General characteristics of subjects}

The subjects' general characteristics, including age, sex, beta-blocker usage, BMI, ejection fraction, and impression, are shown in Table 1. The mean participant age was 55.73 years. The patients were predominantly male, and $85 \%$ of the included patients were receiving beta-blockers. The average ejection fraction was $57.90 \% \pm 8.85 \%$. The ratio of ST elevation and non-ST elevation myocardial infarction was also similar.

Table 1. Subjects' general characteristics

\begin{tabular}{|lc|}
\hline \multicolumn{1}{|c}{ Characteristic } & Value \\
\hline Age $(\mathrm{yr})$ & $55.73 \pm 11.25$ \\
\hline Sex & \\
\hline Male & 53 \\
\hline Female & 7 \\
\hline Beta-blocker usage (\%) & 85 \\
\hline BMI $\left(\mathrm{kg} / \mathrm{m}^{2}\right)$ & $25.13 \pm 3.18$ \\
\hline EF $(\%)$ & $57.90 \pm 8.85$ \\
\hline Impression & \\
\hline STEMI & 31 \\
\hline NSTEMI & 29 \\
\hline Involved coronary vessels & \\
\hline 1 vessel & 45 \\
\hline 2 vessels & 14 \\
\hline 3 vessels & 1 \\
\hline
\end{tabular}

Values are presented as number or mean \pm standard deviation or number.

BMI, body mass index; EF, ejection fraction; STEMI, ST elevation myocardial infarction; NSTEMI, non-ST elevation myocardial infarction.
Variables at 3 weeks after AMI onset and 3 months after first ETT

Post-exercise HRR-0 was a mean $43.70 \pm 14.39$ at baseline and $49.00 \pm 15.51$ after 3 months. Post-exercise HRR3 was $55.39 \pm 14.26$ at baseline and $60.55 \pm 16.59$ after 3 months (Fig. 1). HRR-0 and HRR-3 improved significantly over time ( $\mathrm{p}=0.009, \mathrm{p}=0.014)$. The $\mathrm{VO}_{2 \max }$ was $27.21 \pm 6.31$ $\mathrm{mL} / \mathrm{kg} / \mathrm{min}$ at baseline and $27.96 \pm 6.09 \mathrm{~mL} / \mathrm{kg} / \mathrm{min}$ after 3 months. The $\mathrm{MET}_{\max }$ was $7.77 \pm 1.80$ at baseline and $7.96 \pm 1.76$ after 3 months. There was no significant change in cardiopulmonary exercise capacity $\left(\mathrm{VO}_{2 \max }\right.$ and $\left.\mathrm{MET}_{\max }\right)$ over time $(\mathrm{p}=0.327, \mathrm{p}=0.418)$ (Table 2$)$.

\section{Correlation between HRR and cardiopulmonary exercise capacity}

We performed a Pearson's coefficient correlation to determine whether the HRR at $\mathrm{T} 0$ and $\mathrm{T} 1$ is associated with cardiopulmonary exercise capacity in each test.

HRR-0 at T0 was associated with $\mathrm{VO}_{2 \max }$ at $\mathrm{T} 0$, and this relevance was statistically significant $(\mathrm{r}=0.469, \mathrm{p}<0.001)$. Similar results were seen for $\mathrm{MET}_{\text {max }}(\mathrm{r}=0.469, \mathrm{p}<0.001)$. HRR-3 at T0 was significantly correlated with $\mathrm{VO}_{2 \max }$ at $\mathrm{T} 0$ $(\mathrm{r}=0.392, \mathrm{p}=0.002)$. HRR-0 at T0 was significantly associated with $\mathrm{VO}_{2 \max }$ at $\mathrm{T} 1(\mathrm{r}=0.396, \mathrm{p}=0.002)$. Likewise, similar results were seen for $\mathrm{MET}_{\max }(\mathrm{r}=0.365, \mathrm{p}=0.004)$. There was a significant correlation between HRR-3 at T0 and $\mathrm{VO}_{2 \max }$ at $\mathrm{T} 1(\mathrm{r}=0.289, \mathrm{p}=0.027)$.

There were significant relationships between HRR at T1

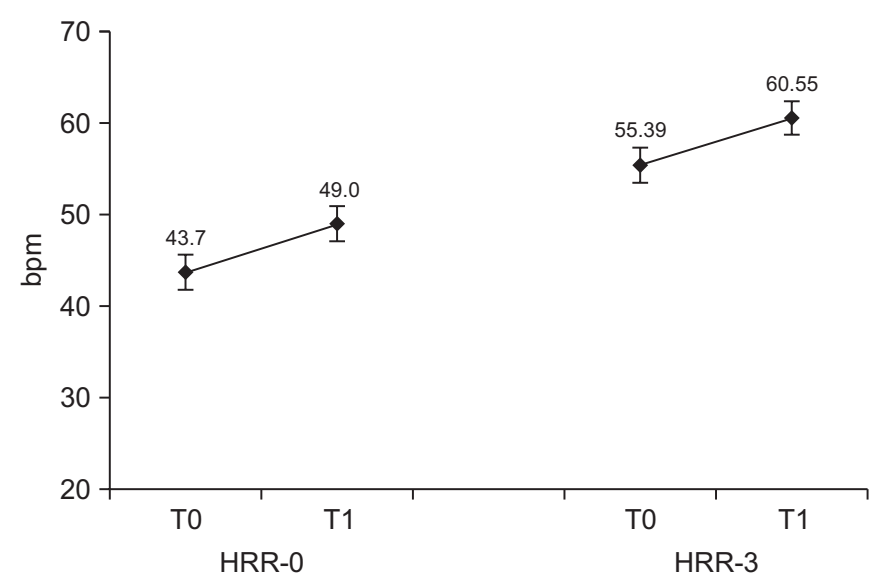

Fig. 1. The heart rate recovery (HRR) at 3 weeks after acute myocardial infarction (T0) and 3 months after first exercise tolerance test (T1). HRR-0, maximal heart rate - heart rate immediately after cool down period; HRR-3, maximal heart rate - heart rate measured 3 minutes after completion of exercise tolerance test. 
Table 2. Variables before and after home-based cardiac rehabilitation

\begin{tabular}{lccc}
\hline & T0 & T1 & p-value \\
\hline $\mathrm{HR}_{\text {rest }}$ (beats/min) & $69.18 \pm 11.03$ & $68.55 \pm 12.86$ & 0.707 \\
$\mathrm{HR}_{\text {max }}$ (beats/min) & $139.25 \pm 18.59$ & $144.90 \pm 19.91$ & $0.007^{*}$ \\
\hline $\mathrm{HRR}-$ (beats/min) & $43.70 \pm 14.39$ & $49.00 \pm 15.51$ & $0.009^{*}$ \\
\hline $\mathrm{HRR}-3$ (beats/min) & $55.39 \pm 14.26$ & $60.55 \pm 16.59$ & $0.014^{*}$ \\
$\mathrm{VO}_{2 \max }(\mathrm{mL} / \mathrm{kg} / \mathrm{min})$ & $27.21 \pm 6.31$ & $27.96 \pm 6.09$ & 0.327 \\
$\mathrm{MET}_{\text {max }}$ & $7.77 \pm 1.80$ & $7.96 \pm 1.76$ & 0.418 \\
$\mathrm{SBP}_{\text {rest }}(\mathrm{mmHg})$ & $114.60 \pm 17.05$ & $119.98 \pm 17.89$ & $0.026^{*}$ \\
$\mathrm{DBP}_{\text {rest }}(\mathrm{mmHg})$ & $74.71 \pm 10.38$ & $76.48 \pm 14.22$ & 0.392 \\
\hline
\end{tabular}

Values are presented as number or mean \pm standard deviation.

T0, exercise tolerance test at 3 weeks after acute myocardial infarction; T1, exercise tolerance test at 3 months after T0; $\mathrm{HR}_{\text {rest }}$, resting heart rate; $\mathrm{HR}_{\text {max }}$, maximal heart rate; $\mathrm{HRR}$, heart rate recovery; HRR-0, maximal heart rate - heart rate immediately after cool down period; HRR-3, maximal heart rate - heart rate measured 3 minutes after completion of exercise tolerance test; $\mathrm{VO}_{2 \max }$, maximal oxygen consumption; $\mathrm{MET}_{\max }$, maximal metabolic equivalents; $\mathrm{SBP}_{\text {rest }}$, resting systolic blood pressure; $\mathrm{DBP}_{\text {rest }}$, resting diastolic blood pressure.

${ }^{*} \mathrm{p}<0.05$.

Table 3. Correlation between HRR and cardiopulmonary exercise capacity

\begin{tabular}{|c|c|c|c|c|c|c|c|c|}
\hline & \multicolumn{2}{|c|}{$\mathrm{VO}_{2 \max }$ at T0 } & \multicolumn{2}{|c|}{ MET $_{\max }$ at T0 } & \multicolumn{2}{|c|}{$\mathrm{VO}_{2 \max }$ at $\mathrm{Tl}$} & \multicolumn{2}{|c|}{ MET $_{\max }$ at T1 } \\
\hline & $\mathbf{r}$ & p-value & $\mathbf{r}$ & p-value & $\mathbf{r}$ & p-value & $\mathbf{r}$ & p-value \\
\hline HRR- 0 at T0 & 0.469 & $0.000^{*}$ & 0.469 & $0.000^{*}$ & 0.396 & $0.002^{*}$ & 0.365 & $0.004^{*}$ \\
\hline HRR-3 at T0 & 0.392 & $0.002^{*}$ & 0.392 & $0.002^{*}$ & 0.289 & $0.027^{*}$ & 0.414 & $0.001^{*}$ \\
\hline HRR- 0 at T1 & - & - & - & - & 0.694 & $0.000^{*}$ & 0.709 & $0.000^{*}$ \\
\hline HRR-3 at T1 & - & - & - & - & 0.668 & $0.000^{*}$ & 0.650 & $0.000^{*}$ \\
\hline
\end{tabular}

HRR, heart rate recovery; T0, exercise tolerance test at 3 weeks after acute myocardial infarction; T1, exercise tolerance test at 3 months after T0; $\mathrm{VO}_{2 \max }$, maximal oxygen consumption; $\mathrm{MET}_{\max }$, maximal metabolic equivalents; HRR-0, maximal heart rate - heart rate immediately after cool down period; HRR-3, maximal heart rate - heart rate measured 3 minutes after completion of exercise tolerance test.

${ }^{*} \mathrm{p}<0.05$.

Table 4. Correlation between HRR at T0 and changing ratio of cardiopulmonary exercise capacity

\begin{tabular}{|c|c|c|c|c|}
\hline & \multicolumn{2}{|c|}{ Changing ratio of $\mathrm{VO}_{2 \max }$} & \multicolumn{2}{|c|}{ Changing ratio of $\mathrm{MET}_{\max }$} \\
\hline & $\mathbf{r}$ & p-value & $\mathbf{r}$ & p-value \\
\hline HRR-0 at T0 & -0.091 & 0.491 & -0.168 & 0.199 \\
\hline HRR-3 at T0 & -0.117 & 0.377 & -0.186 & 0.159 \\
\hline HRR-0 at T1 & 0.364 & $0.004^{*}$ & 0.382 & $0.002^{*}$ \\
\hline HRR-3 at T1 & 0.379 & $0.003^{*}$ & 0.350 & $0.006^{*}$ \\
\hline
\end{tabular}

HRR, heart rate recovery; T0, exercise tolerance test at 3 weeks after acute myocardial infarction; T1, exercise tolerance test at 3 months after T0; $\mathrm{VO}_{2 \max }$, maximal oxygen consumption; $\mathrm{MET}_{\max }$, maximal metabolic equivalents; HRR-0, maximal heart rate - heart rate immediately after cool down period; HRR-3, maximal heart rate - heart rate measured 3 minutes after completion of exercise tolerance test.

Changing ratio of $\mathrm{VO}_{2 \max }=\left(\mathrm{VO}_{2 \max }\right.$ at $\mathrm{T} 1-\mathrm{VO}_{2 \max }$ at $\left.\mathrm{T} 0\right) / \mathrm{VO}_{2 \max }$ at $\mathrm{T} 0 \times 100$.

Changing ratio of $\mathrm{MET}_{\max }=\left(\mathrm{MET}_{\max }\right.$ at $\mathrm{T} 1-\mathrm{MET}_{\max }$ at $\left.\mathrm{T} 0\right) / \mathrm{MET}_{\max }$ at $\mathrm{T} 0 \times 100$.

${ }^{*} \mathrm{p}<0.05$. 
and cardiopulmonary exercise capacity at $\mathrm{T} 1$ (Table 3).

\section{Correlation between HRR at To and changing \\ cardiopulmonary exercise capacity ratio}

Both HRR-0 and HRR-3 at T0 were not correlated with the changing ratio of $\mathrm{VO}_{2 \max }(\mathrm{r}=-0.091, \mathrm{p}=0.491$ and $\mathrm{r}=$ $-0.117, p=0.377$, respectively). Neither HRR-0 nor HRR3 at T0 was correlated with the changing ratio of $\mathrm{MET}_{\max }$ $(\mathrm{r}=0.168, \mathrm{p}=0.199$ and $\mathrm{r}=-0.186, \mathrm{p}=0.159$, respectively) (Table 4). These results were similar in a multivariate regression analysis. In the multivariate regression analysis, both HRR-0 and HRR-3 at T0 did not show independent prognostic value for the changing ratio of cardiopulmonary exercise capacity when controlled for age, BMI, EF, $\mathrm{HR}_{\text {max }}, \mathrm{VO}_{2 \max }, \mathrm{MET}_{\max }$. The stepwise method multivariate regression analysis also did not indicate an independent association of both HRR- 0 and HRR-3 with the changing ratio of cardiopulmonary exercise capacity after adjusting the variables mentioned above (Table 5).

\section{DISCUSSION}

Here we attempted to elucidate the relationship between functional capacity and autonomic function. A significant association has been reported between HRR and peak $\mathrm{VO}_{2}$ to date. Sheppard et al. [9] reported that HRR after a cardiopulmonary exercise test was correlated with peak $\mathrm{VO}_{2}$ in patients with heart failure. Bilsel et al. [13] demonstrated that patients with an abnormal HRR had a lower $\mathrm{VO}_{2 \max }$ value than those with a normal HRR. Similarly to other studies, this study demonstrated that HRR is correlated with cardiopulmonary exercise capacity. Unlike other studies, this study showed that HRR at T0 is also significantly related with cardiopulmonary exercise capacity at $\mathrm{T} 1$. In other words, subjects with a lower baseline HRR would have lower functional capacity, even after a few months.

The main purpose of this study was to determine the relationship between autonomic function and changes in functional capacity in patients with AMI. We hypothesized that there would be a relationship between the two. The study showed a significant relationship between HRR at T0 and exercise capacity at T0 as well as between HRR at $\mathrm{T} 0$ and exercise capacity at $\mathrm{T} 1$, but not between HRR at $\mathrm{T} 0$ and a changing ratio in functional capacity. Therefore, these findings may be interpreted as reflecting the initial severity rather than the direct impact of the changes in exercise capacity.

Youn et al. [11] reported that Post-exercise HRR is an index of autonomic function associated with clinical outcomes in patients with heart disease, and the increase in HRR represents a positive adaptation of autonomic function. This study showed that the autonomic response can improve over time. However, increases in exercise capacity were not significant. That is, the passage of time did not increase cardiopulmonary exercise capacity on its own without intervention, such as with exercise-based cardiac rehabilitation. Several studies have shown that cardiac rehabilitation improves physical health and exercise capacity in patients with coronary heart disease [1719]. Therefore, another noteworthy point in our study is that interventions such as exercise-based cardiac rehabilitation may be necessary to improve cardiopulmonary exercise capacity.

We attempted to clarify whether a change in exercise capacity after a few months can be predicted in relation to autonomic dysfunction upon onset of AMI. Unfortunately, we have not proven the hypothesis we established in this study. However, it is meaningful that we reported the relationship between HRR at initial ETT and exercise capacity after three months after first ETT, unlike other studies that have reported the relationship between HRR and exercise capacity only at one time point. In addition, this study is significant in that it clarified the change over time of autonomic dysfunction after AMI. There was a previous study of autonomic function improvement by active center-based cardiac rehabilitation, but few studies have investigated the natural course of autonomic function [20].

Our study has some limitations. First, it was retrospective, so the results may have been affected by its observational nature. The potential for referral bias exists because this report selected data from an exercise test database. However, to reduce as much intervention bias as possible, this study excluded patients who underwent a center-based cardiac rehabilitation program to enable an investigation of the natural course on the relationship between HRR and cardiopulmonary exercise capacity. However, it should be noted in interpreting this result that although this study targeted patients who did not participate in a center-based cardiac rehabilitation program, patient education was performed in the same way 


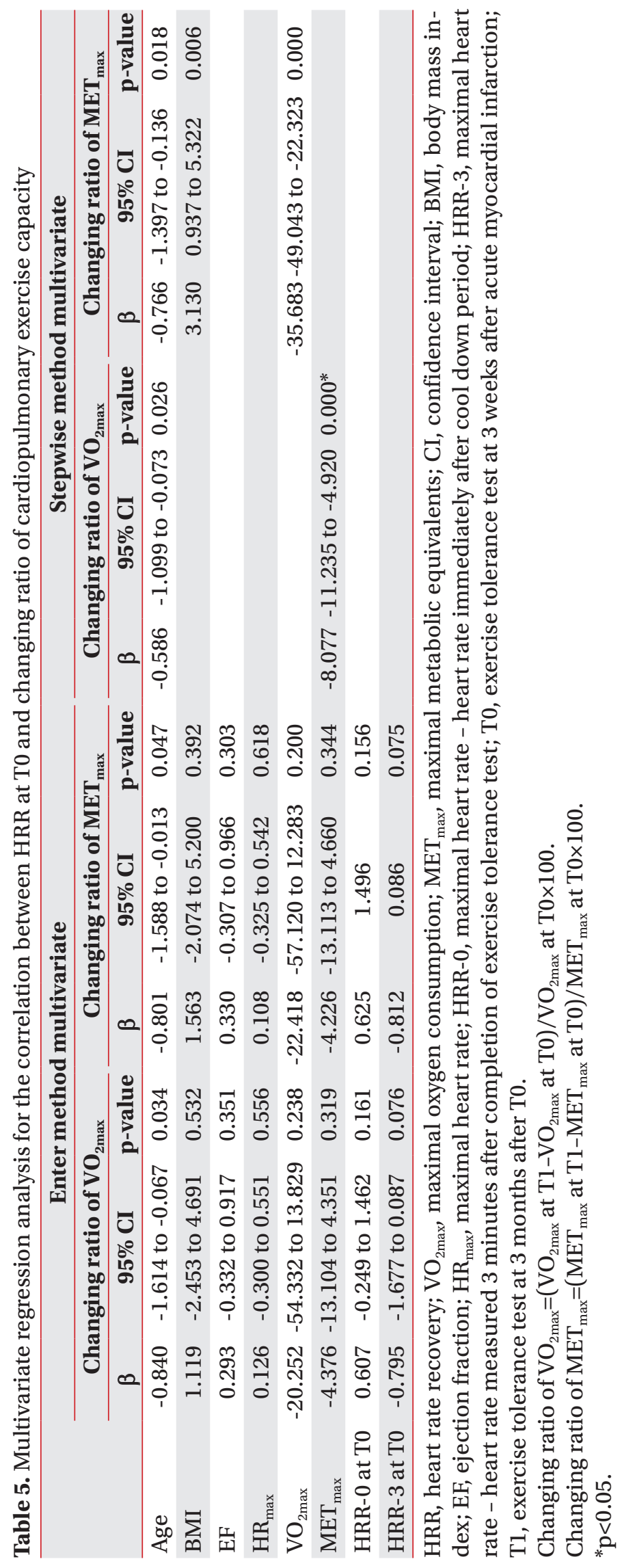


at every visit. Second, the sample size was small due to the cost of follow-up ETT, patients' lack of motivation, and patients' difficult hospital accessibility. Third, other factors such as beta-blocker use may have influenced autonomic function, and we did not consider such factors in our analysis. However, studies have demonstrated that beta-blockers were not associated with post-exercise HRR and did not modify the association between HRR and clinical outcomes $[2,9,11]$. Finally, since the exercise intensity, time, frequency, and duration of each patient was uncontrolled, there might be a difference in the degree of adaptation of autonomic function according to exercise amount. Future studies including formalized cardiac rehabilitation protocols are desirable.

In conclusion, this study shows that post-exercise HRR is associated with functional capacity as well as clinical outcomes. However, this measurement could not establish the prognostic value in relation to the degree of cardiopulmonary exercise capacity enhancement.

\section{CONFLICT OF INTEREST}

No potential conflict of interest relevant to this article was reported.

\section{ACKNOWLEDGMENTS}

This work was supported by the National Research Foundation of Korea (NRF) with a grant funded by the Korean government (MSIP) (No. 2016R1C1B2016178).

\section{REFERENCES}

1. Olshansky B, Sabbah HN, Hauptman PJ, Colucci WS. Parasympathetic nervous system and heart failure: pathophysiology and potential implications for therapy. Circulation 2008;118:863-71.

2. Tang YD, Dewland TA, Wencker D, Katz SD. Postexercise heart rate recovery independently predicts mortality risk in patients with chronic heart failure. J Card Fail 2009;15:850-5.

3. Vivekananthan DP, Blackstone EH, Pothier CE, Lauer MS. Heart rate recovery after exercise is a predictor of mortality, independent of the angiographic severity of coronary disease. J Am Coll Cardiol 2003;42:831-8.

4. Nishime EO, Cole CR, Blackstone EH, Pashkow FJ,
Lauer MS. Heart rate recovery and treadmill exercise score as predictors of mortality in patients referred for exercise ECG. JAMA 2000;284:1392-8.

5. Cole CR, Blackstone EH, Pashkow FJ, Snader CE, Lauer MS. Heart-rate recovery immediately after exercise as a predictor of mortality. N Engl J Med 1999;341: 1351-7.

6. Imai K, Sato H, Hori M, Kusuoka H, Ozaki H, Yokoyama $\mathrm{H}$, et al. Vagally mediated heart rate recovery after exercise is accelerated in athletes but blunted in patients with chronic heart failure. J Am Coll Cardiol 1994;24:1529-35.

7. Lipinski MJ, Vetrovec GW, Gorelik D, Froelicher VF. The importance of heart rate recovery in patients with heart failure or left ventricular systolic dysfunction. J Card Fail 2005;11:624-30.

8. Arena R, Guazzi M, Myers J, Peberdy MA. Prognostic value of heart rate recovery in patients with heart failure. Am Heart J 2006;151:851.

9. Sheppard RJ, Racine N, Roof A, Ducharme A, Blanchet $M$, White M. Heart rate recovery: a potential marker of clinical outcomes in heart failure patients receiving beta-blocker therapy. Can J Cardiol 2007;23:1135-8.

10. Kubrychtova V, Olson TP, Bailey KR, Thapa P, Allison TG, Johnson BD. Heart rate recovery and prognosis in heart failure patients. Eur J Appl Physiol 2009;105:3745.

11. Youn JC, Lee HS, Choi SW, Han SW, Ryu KH, Shin EC, et al. Post-exercise heart rate recovery independently predicts clinical outcome in patients with acute decompensated heart failure. PLoS One 2016;11: e0154534.

12. Karjalainen JJ, Kiviniemi AM, Hautala AJ, Piira OP, Lepojarvi ES, Peltola MA, et al. Determinants and prognostic value of cardiovascular autonomic function in coronary artery disease patients with and without type 2 diabetes. Diabetes Care 2014;37:286-94.

13. Bilsel T, Terzi S, Akbulut T, Sayar N, Hobikoglu G, Yesilcimen $\mathrm{K}$. Abnormal heart rate recovery immediately after cardiopulmonary exercise testing in heart failure patients. Int Heart J 2006;47:431-40.

14. Cohn JN, Johnson GR, Shabetai R, Loeb H, Tristani $\mathrm{F}$, Rector T, et al. Ejection fraction, peak exercise oxygen consumption, cardiothoracic ratio, ventricular arrhythmias, and plasma norepinephrine as determinants of prognosis in heart failure. The V-HeFT 
VA Cooperative Studies Group. Circulation 1993;87(6 Suppl):VI5-16.

15. Passantino A, Scrutinio D, Lagioia R, Forleo C, Ricci A, Rizzon P. Value of peak oxygen consumption during exercise for the prognostic stratification of patients with severe systolic dysfunction of the left ventricle. Cardiologia 1997;42:947-52.

16. Metra M, Faggiano P, D'Aloia A, Nodari S, Gualeni A, Raccagni $\mathrm{D}$, et al. Use of cardiopulmonary exercise testing with hemodynamic monitoring in the prognostic assessment of ambulatory patients with chronic heart failure. J Am Coll Cardiol 1999;33:943-50.

17. Jolliffe JA, Rees K, Taylor RS, Thompson D, Oldridge N, Ebrahim S. Exercise-based rehabilitation for coronary heart disease. Cochrane Database Syst Rev 2001;1:
CD001800.

18. Taylor RS, Brown A, Ebrahim S, Jolliffe J, Noorani H, Rees K, et al. Exercise-based rehabilitation for patients with coronary heart disease: systematic review and meta-analysis of randomized controlled trials. Am J Med 2004;116:682-92.

19. Heran BS, Chen JM, Ebrahim S, Moxham T, Oldridge $\mathrm{N}$, Rees K, et al. Exercise-based cardiac rehabilitation for coronary heart disease. Cochrane Database Syst Rev 2011;7:CD001800.

20. Lazzeroni D, Castiglioni P, Bini M, Faini A, Camaiora U, Ugolotti PT, et al. Improvement in aerobic capacity during cardiac rehabilitation in coronary artery disease patients: is there a role for autonomic adaptations? Eur J Prev Cardiol 2017;24:357-64. 